Taxonomía y sistemática

\title{
Primer registro de un estípite de Coryphoideae: Palmoxylon kikaapoa de la Formación Olmos del Cretácico Superior, Coahuila, México
}

\section{First record of a palm trunk Coryphoideae: Palmoxylon kikaapoa from the Olmos Formation of the Upper Cretaceous, Coahuila, Mexico}

\author{
B. Alejandra Sainz-Resendiz ${ }^{a}$, Emilio Estrada-Ruiz ${ }^{\text {b* }}$, L. Elena Mateo-Cid ${ }^{\mathrm{c}}$ \\ y Héctor Porras-Múzquiz ${ }^{\mathrm{d}}$
}

${ }^{a}$ Maestría en Biociencias, Departamento de Zoología, Escuela Nacional de Ciencias Biológicas, Instituto Politécnico Nacional, Prolongación de Carpio y Plan de Ayala s/n, 11340 México, D.F., México

${ }^{\mathrm{b}}$ Laboratorio de Ecología, Departamento de Zoología, Escuela Nacional de Ciencias Biológicas, Instituto Politécnico Nacional, Prolongación de Carpio y Plan de Ayala s/n, 11340 México, D.F., México

${ }^{\mathrm{c}}$ Laboratorio de Ficología, Departamento de Botánica, Escuela Nacional de Ciencias Biológicas, Instituto Politécnico Nacional, Prolongación de Carpio y Plan de Ayala s/n, 11340 México, D.F., México

d Museo de Múzquiz, A. C., Zaragoza 209, 26340 Múzquiz, Coahuila, México

Recibido el 23 de febrero de 2015; aceptado el 11 de agosto de 2015

Disponible en Internet el 6 de noviembre de 2015

\section{Resumen}

En sedimentos de la Formación Olmos del Cretácico Tardío (Campaniano superior) se ha recolectado una flora diversa representada por flores, frutos, hojas y maderas de angiospermas, gimnospermas y polypodiales, que documenta una flora de tipo paratropical. Del material recolectado recientemente, se da a conocer una especie fósil nueva de palma, asignada a Palmoxylon kikaapoa. El estípite permineralizado se caracteriza por presentar un atactostela con haces fibrovasculares simples de forma reniforme; 1-4 vasos del metaxilema con placa de perforación escalariforme y oblicua; 1-5 elementos de protoxilema con placa de perforación simple; parénquima paravascular desarrollado; floema en un solo conjunto; parénquima tabular con 2-8 capas de células alargadas; tejido fundamental aerenquimatoso tipo A; numerosos haces fibrosos en el tejido fundamental; estegmatas esféricas en filas discontinuas en la periferia de las fibras de los haces fibrovasculares y fibrosos. Estos caracteres anatómicos permitieron relacionarla taxonómicamente con la familia Arecaceae, subfamilia Coryphoideae y tribu Trachycarpeae. Tomando en cuenta los antecedentes paleobotánicos y botánicos, se infiere que la especie fósil se desarrolló bajo un clima tropical con un hábito de vida de tipo arbóreo.

Derechos Reservados (C) 2015 Universidad Nacional Autónoma de México, Instituto de Biología. Este es un artículo de acceso abierto distribuido bajo los términos de la Licencia Creative Commons CC BY-NC-ND 4.0.

Palabras clave: Anatomía; Arecaceae; Campaniano superior; Subfamilia Coryphoideae; Trachycarpeae

\begin{abstract}
In sediments of the Olmos Formation from the Late Cretaceous (upper Campanian) a diverse flora represented by flowers, fruits, leaves and wood of angiosperms, gymnosperms and polypodiales has been collected, these records document the presence of a flora with paratropical affinities. Here we describe a new fossil palm species, Palmoxylon kikaapoa. The permineralized palm trunk is characterized by an atactostele with fibro-vascular bundles simple with reniform shape; with 1-4 vessels of metaxylem with scalariform and oblique perforation plates; 1-5 protoxylem elements with simple perforation plates; paravascular parenchyma developed; phloem in one phloem strad; tabular parenchyma with 2-8 layers of elongated cells; ground tissue consisting of thin-walled cells forming a not compact tissue with intercellular spaces, type A; numerous fibrous bundles in the
\end{abstract}

\footnotetext{
* Autor para correspondencia.

Correo electrónico: emilkpaleobot@yahoo.com.mx (E. Estrada-Ruiz).

La revisión por pares es responsabilidad de la Universidad Nacional Autónoma de México.
} 
fundamental tissue; spherical stegmata occur in discontinuous files next to fibro-vascular bundles or fibrous bundles. These anatomical characters allowed us to taxonomically relate the fossil to the family Arecaceae, subfamily Coryphoideae, and tribe Trachycarpeae. Taking into account paleobotanical and botanical evidence, we propose a tropical affinity and an arboreal habit for this fossil species.

All Rights Reserved (c) 2015 Universidad Nacional Autónoma de México, Instituto de Biología. This is an open access item distributed under the Creative Commons CC License BY-NC-ND 4.0.

Keywords: Anatomy; Arecaceae; Upper Campanian; Subfamily Coryphoideae; Trachycarpeae

\section{Introducción}

En el estado de Coahuila, en el área de Monclova-Sabinas, se localiza la Cuenca de Sabinas, dentro de ella se encuentran los sedimentos de la Formación Olmos del Cretácico tardío (Campaniano superior; Estrada-Ruiz, Martínez-Cabrera, Callejas-Moreno y Upchurch, 2013). Esta formación geológica es importante económicamente por la extracción de carbón vegetal, además, tiene gran interés científico por la abundancia de plantas fósiles que alberga. Un estudio paleoclimático basado en la fisonomía foliar de angiospermas indica que esta paleoflora creció en un clima de tipo paratropical $\left(20-25^{\circ} \mathrm{C}\right.$ ) (Estrada-Ruiz, Upchurch y Cevallos-Ferriz, 2008). La flora, cuyo componente taxonómico principal son las angiospermas, tiene una alta tasa de endemismos genéricos, sin embargo, existen también otros géneros que tuvieron una distribución más amplia, encontrándose hasta el centro-sur de EE. UU. como lo denota el registro de madera de Javelinoxylon Wheeler, Lehman y Gasson encontrado en Texas (EE. UU.), Coahuila (México) y recientemente en Chihuahua (México) (Estrada-Ruiz, Martínez-Cabrera y Cevallos-Ferriz, 2007, 2010; Estrada-Ruiz y Martínez-Cabrera, 2011; Weber, 1972, 1978; Wheeler, Lehman y Gasson, 1994). A pesar de que se han recolectado más de 100 morfotipos de angiospermas, el número descrito hasta la fecha no supera el $30 \%$ del material identificado formalmente (e.g., Estrada-Ruiz et al., 2007; Estrada-Ruiz, Martínez-Cabrera y Cevallos-Ferriz, 2010; Estrada-Ruiz et al., 2013; Estrada-Ruiz, Upchurch, Wolfe y Cevallos-Ferriz, 2011; Weber, 1972, 1978).

Weber (1972) inició formalmente el estudio de esta flora a principios de la década de 1970, donde menciona la presencia de al menos 80 especies fósiles con base en flores, frutos, hojas y maderas de angiospermas y gimnospermas. Se han descrito formalmente algunas monilofitas representadas por helechos de formas acuáticas como Salvinia Ség. y Dorfiella Weber (Weber, 1973, 1976), además de al menos otros 5 a 7 miembros de polypodiales. Adicionalmente, se han encontrado preservadas como estructuras reproductivas y vegetativas de conos, brácteas y maderas relacionadas con coníferas (Cevallos-Ferriz, 1992; Serlin, Delevoryas y Weber, 1981; Weber, 1975); en particular a las maderas de coníferas descritas para la Formación Olmos se las ha relacionado con Podocarpaceae y Taxodiaceae (CevallosFerriz, 1992).

Se han descrito hojas de angiospermas con afinidad a Arecaceae, Magnoliaceae, Araceae, Palmae, Lauraceae, Nelumbonaceae y diversas Laurales, y una inflorescencia de Arecaceae comparable con el género Manicaria Gaertn (Estrada-Ruiz et al., 2008; Estrada-Ruiz et al., 2011; Weber, 1972, 1978). También se han documentado maderas de angiospermas relacionadas con Lauraceae, Fagaceae, Malvaceae s.l., Cornaceae, Anacardiaceae/Burseraceae, Ericales y Metcalfeoxylon Wheeler, McClammer y LaPasha (Cevallos-Ferriz y Weber, 1992; Estrada-Ruiz et al., 2007, 2010), y se han reconocido estípites de monocotiledóneas asignadas a varias especies del género fósil Palmoxylon Schenk, como P. longum CevallosFerriz y Ricalde-Moreno, $P$. commune Cevallos-Ferriz y Ricalde-Moreno, P. fibrosum Cevallos-Ferriz y Ricalde-Moreno, P. polymorphum Cevallos-Ferriz y Ricalde-Moreno y P. enochii Estrada-Ruiz y Cevallos-Ferriz (Cevallos-Ferriz y RicaldeMoreno, 1995; Estrada-Ruiz y Cevallos-Ferriz, 2009). Todos estos registros claramente denotan que la flora de la Formación Olmos, además de ser muy diversa, se desarrolló en un ambiente tropical (Estrada-Ruiz et al., 2008; Estrada-Ruiz et al., 2013).

Respecto a la taxonomía de las palmas fósiles, estas presentan una gran variabilidad anatómica en sus órganos, por lo que frecuentemente se limita la posibilidad de inferir afinidades taxonómicas más finas como a nivel género o tribu. Esto ha conducido que al describir sus tallos solo sean incluidos en un único género fósil conocido como Palmoxylon (Harley, 2006). En EE. UU. se han descrito no más de 10 especies cretácicas. El registro fósil más viejo documentado para EE. UU. de Palmoxylon corresponde a $P$. cliffwoodensis del Coniaciano-Santoniano de Nueva Jersey (Berry, 1916). Respecto al registro fósil de la subfamilia Trachycarpeae, se han descrito formalmente no más de 12 especies fósiles (Dransfield et al., 2008); específicamente sobre el género Copernicia, su registro fósil es muy escaso. Macko (1957) recolectó polen en sus investigaciones sobre la flora del Mioceno Inferior de Polonia (Dransfield et al., 2008). Brea, Zucol y Franco (2013) describieron fitolitos de este género de la Formación Paraná (Mioceno tardío) de Argentina.

En este trabajo se describe el primer estípite para el Cretácico Superior de la subfamilia Coryphoideae, tribu Trachycarpeae para México. Con este nuevo registro de Palmoxylon se apoya aún más la hipótesis de que durante este periodo las palmas fueron muy abundantes y diversas y conformaron un componente importante en ambientes cercanos a cuerpos de agua, además, algunas de ellas formaron parte del estrato arbóreo dentro de la flora de la Formación Olmos.

La Formación Olmos representa un sistema deltaico-fluvial (Estrada-Ruiz, 2009). La formación tiene representados 4 subambientes de depósito. El primero corresponde a la litofacies A, representada por carbón tipo subbituminoso, que sugiere un ambiente de depósito en zonas pantanosas de circulación restringida. La segunda representa la litofacies B, compuesta de lutitas y areniscas que puede representar ambientes de planicies de inundación y/o lagunas con circulación abierta. La litofacies $\mathrm{C}$, corresponde a un ambiente fluvial probablemente de ríos 


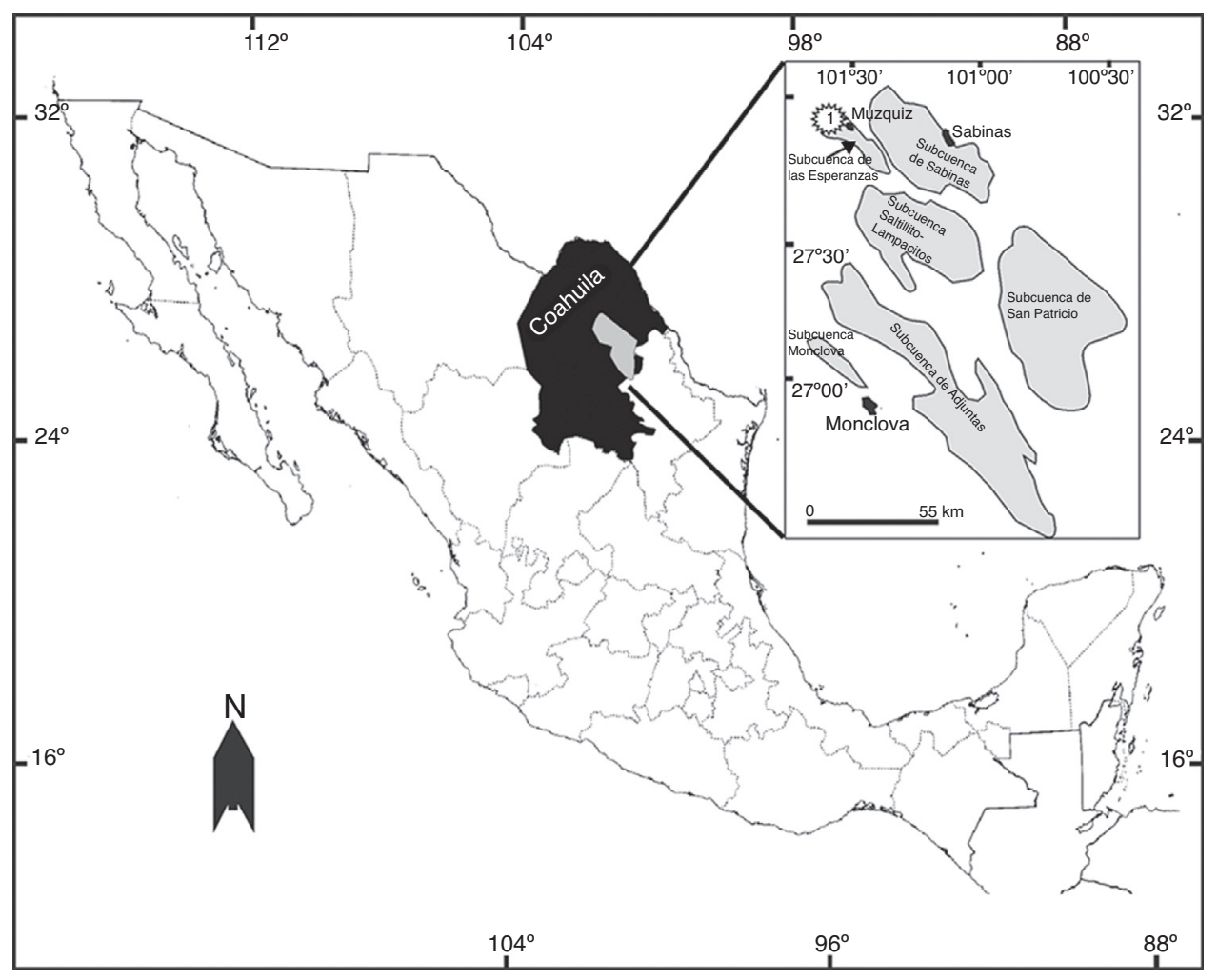

Figura 1. Área de estudio. 1, rancho Atascoso, a 5 km de la población de Múzquiz, Coahuila, México (modificada de Estrada-Ruiz y Cevallos-Ferriz, 2009).

entrelazados, como lo sugiere la geometría de las barras arenosas y rellenos de canales; por último, las areniscas con estratificación cruzada que pertenece a la litofacies $\mathrm{D}$, pueden interpretarse como relleno de canales y barras laterales depositadas en un ambiente de ríos meándricos.

Todas las maderas y estípites fósiles que se han estudiado hasta la fecha provienen del sistema fluvial representado por ríos entrelazados y subambientes con ríos meandrosos pertenecientes a la litofacies D (Estrada-Ruiz et al., 2007, 2010; Estrada-Ruiz y Cevallos-Ferriz, 2009).

\section{Materiales y métodos}

El estípite permineralizado se recolectó en el año 2012 en sedimentos de la Formación Olmos, en la localidad conocida como Rancho Atascoso (2753’35.7” N, 101 34’30” O), al noroeste del poblado de Múzquiz, municipio de Melchor Múzquiz, Coahuila. En esta misma área se han recolectado otros tipos de maderas representados principalmente por angiospermas y coníferas (Estrada-Ruiz et al., 2010). Todas las muestras se han encontrado en forma de rodados y algunas de ellas alcanzaron hasta $2 \mathrm{~m}$ de longitud con $50 \mathrm{~cm}$ de diámetro (Estrada-Ruiz et al., 2013) (fig. 1).

Para la descripción e identificación taxonómica del estípite se realizaron cortes transversales y longitudinales con la técnica de lámina delgada (Haas y Rowe, 1999); para el corte de los tallos fósiles se utilizó una cortadora marca Buhler, con disco de punta de diamante marca Buhler. El espesor de cada laminilla fue de $30 \mu \mathrm{m}$, aproximadamente. Para la observación y cuantificación de los caracteres anatómicos de la palma se utilizó un microscopio óptico Zeiss-Axiostar con una cámara digital AxioCam MRc de Zeiss, para las tomas de las microfotografías, con la finalidad de mostrar gráficamente los datos obtenidos.

Se realizaron 25 mediciones para cada carácter anatómico. Para la identificación del fósil se revisó literatura especializada en el tema (e.g., Cevallos-Ferriz y Ricalde-Moreno, 1995; Estrada-Ruiz y Cevallos-Ferriz, 2009; Sahni, 1964; Tidwell, Medlyn y Thyan, 1972; Tomlinson, Horn y Fisher, 2011; Uhl y Dransfield, 1987; Weiner y Liese, 1993). Además, se consultó la base de datos electrónica Palmweb, 2011 (www.palmweb.org) para la identificación, así como para conocer las distribuciones de algunos taxones actuales (Thomas y de Franceschi, 2012, 2013); Palm-ID es una base de datos interactiva empleada para identificar a las palmas con base en la anatomía del tallo empleando el sistema $\left(\mathrm{Xper}^{2}\right)$ que es actualizado continuamente (Thomas, 2011; URL http://www.infosyslab.fr/Palm-id/; 2014).

El estípite permineralizado se encuentra resguardado en la Colección de Paleontología del Museo de Múzquiz, A.C., Múzquiz, Coahuila. El tallo está precedido por la abreviatura MUZ-1336, además, a cada lámina delgada se le asignó un número, los mismos que se utilizaron para ilustrar en cada pie de figura. Para su clasificación taxonómica se empleó APG III (Angiosperm Phylogeny Group, 2009). Se utilizaron los nombres taxonómicos propuestos por Chase y Reveal (2009), complementados con nombres para clados bien corroborados por datos moleculares. 

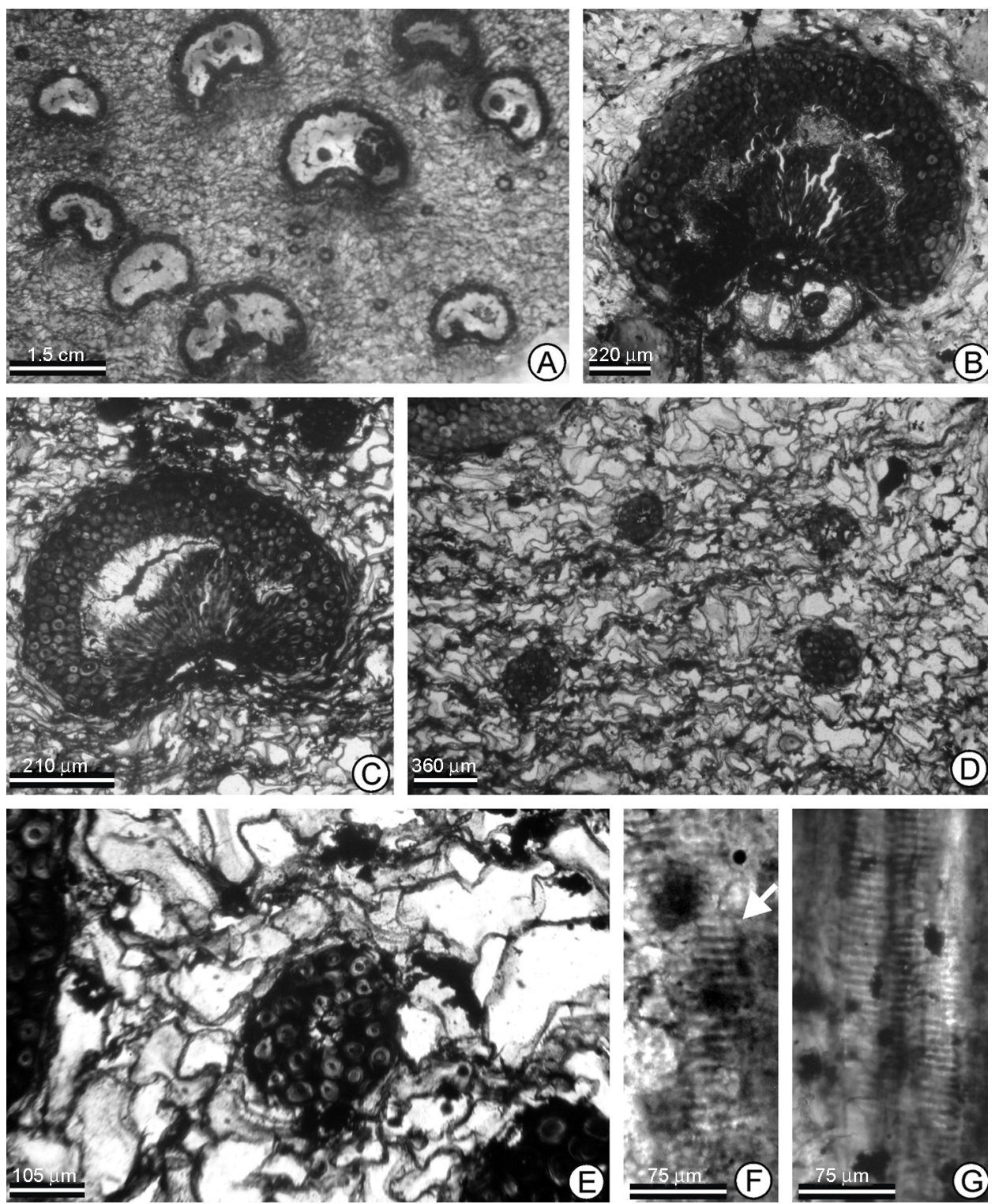

Figura 2. A-G. Descripción anatómica de Palmoxylon kikaapoa sp. nov. A, corte transversal (CT) haces vasculares orientados al azar, presenta 2 tipos de tamaños; B, (CT) un haz vascular, mostrando 4 vasos del metaxilema; C, (CT) un haz vascular, mostrando 2 vasos del metaxilema; D, (CT) vista general del tejido fundamental con la presencia de fibras; E, (CT) detalle del tejido fundamental y un casquete de fibras; F, (CL) vaso con placa de perforación escalariforme (flecha); G, (CL) 2 elementos de vaso con punteaduras opuestas a escalariformes.

\section{Resultados}

Familia Arecaceae Schultz Sch. 1832

Subfamilia Coryphoideae Burnett, 1835

Tribu Trachycarpeae

Género Palmoxylon Schenk, 1882

Especie tipo. Palmoxylon blanfordii Schenk, 1882

Palmoxylon kikaapoa sp. nov. (figs. $2 \mathrm{~A}-\mathrm{G}$ y $3 \mathrm{~A}-\mathrm{G}$ )

\section{Diagnosis}

Porción distal del estípite con atactostela. Haces fibrovasculares simples arreglados al azar, de forma reniforme y en quilla; 1-4 elementos de metaxilema con placa de perforación escalariforme y oblicua con punteaduras opuestas a escalariformes, 1-5 elementos de protoxilema con placa de perforación simple; parénquima paravascular desarrollado; floema en un solo conjunto; parénquima tabular con 2 a 8 capas de células alargadas; tejido fundamental aerenquimatoso tipo A; numerosos haces fibrosos en el tejido fundamental; estegmatas esféricas en filas discontinuas.

Distal portion of the stem with atactostele. Simple vascular bundles randomly arranged, reniform and keel-shaped; each vascular bundle with 1 to 4 metaxylem vessels with scalariform perforation plates and scalariform to opposite pits; 1 to 5 protoxylem cells with simple perforation plates; paravascular parenchyma developed; phloem in a single set; tabular parenchyma with 2-8 layers of elongated cells in cross-section; type 

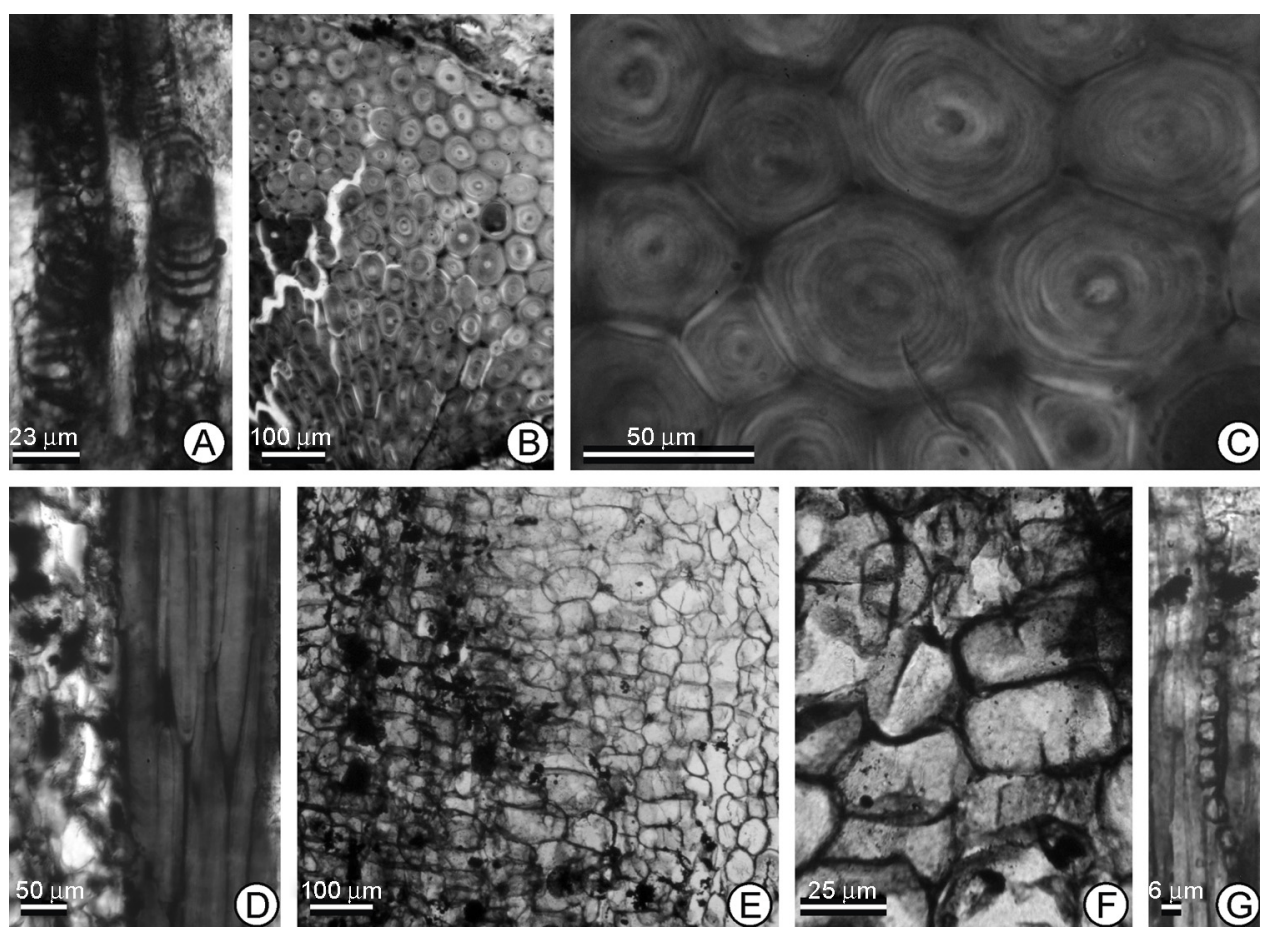

Figura 3. A-G. Descripción anatómica de Palmoxylon kikaapoa sp. nov. A, corte longitudinal (CL) engrosamiento anular de los vasos del protoxilema; B, corte transversal (CT) detalle de las células de las fibras de un haz fibrovascular; C, (CT) detalle de las fibras del haz fibrovascular, se observan los engrosamientos plurilaminados; D, (CL) detalle de las fibras que se encuentran en el tejido fundamental; E, (CL) células del tejido fundamental; F, (CL) detalle de las células isodiamétricas del tejido fundamental; G, (CL) detalle de las estegmatas.

A aerenchyma in ground tissue; numerous fiber bundles in ground tissue; spherical stegmata in discontinuous rows.

\section{Descripción}

Porción distal del estípite pequeña, en atactostela. Se observa el tejido fundamental y haces fibrovasculares (fig. 2A-D). Los haces fibrovasculares presentan un promedio de 16 (13-19) por $\mathrm{cm}^{2}$ (fig. 2A). Estos tienen forma reniforme (fig. 2A, B) y en quilla, con un área vascular y otra fibrosa, en una proporción de 1:3 (fig. 2A-C). El área vascular con 236 (103-415) $\mu \mathrm{m}$ de alto y 437 (256-560) $\mu \mathrm{m}$ de ancho (fig. 2B). El xilema tiene usualmente 2 a 3 vasos de metaxilema, pero algunas veces está formado hasta por 4 (fig. 2B, C). Los elementos de vaso del metaxilema en corte transversal tienen forma redonda a oval. Su diámetro promedio es de 74 (42-119) $\mu \mathrm{m}$, con punteaduras opuestas a escalariformes (fig. 2G) y placa de perforación escalariforme y oblicua (fig. 2F). En algunas zonas vasculares se observan de 1-5 elementos traqueales del protoxilema, de forma circular a oval con un diámetro promedio de $23(18-27) \mu \mathrm{m}$, tienen engrosamientos anulares a helicoidales, con placa de perforación simple (fig. 3A). Los elementos conductores de los haces fibrovasculares están rodeados por parénquima paravascular desarrollado con pared delgada (fig. 2A-C) y a su vez rodeado por células de parénquima de forma tabular formado por 2 a 3 células de forma rectangular y con pared delgada (fig. 2B, C). Entre el área vascular y el área fibrosa hay un espacio de forma cóncava, que corresponde al área donde estaba el floema en un solo conjunto.
Rodeando parcialmente al área vascular, se encuentra el área fibrosa sin elongación radial. Su diámetro promedio es de 1,127 $(828-1,541) \mu \mathrm{m}$ de alto y $1,436(881-1,918) \mu \mathrm{m}$ de ancho, en estas mediciones se incluye al área vascular. Está constituida por fibras plurilaminadas arregladas de forma compacta (fig. 3B, C), las fibras de menor diámetro se localizan en la parte central del fascículo, limitante con el área vascular, mientras que las de mayor diámetro se localizan en la periferia, el diámetro tangencial promedio de la luz es de 7 (2-22) $\mu \mathrm{m}$ y la pared de 49 (20-79) $\mu \mathrm{m}$ (fig. 3B, C).

En el tejido fundamental se observan 2 tipos de células. El parénquima tabular rodea a los haces fibrovasculares formando 2 a 8 capas, compuesto por células alargadas de pared delgada (fig. 2A-C). El segundo tipo forma el resto del tejido fundamental, con tendencia a ser aerenquimatoso; en corte transversal sus células se organizan en un tejido no compacto, las células están poco ramificadas dejando espacios intercelulares redondeados e irregulares entre ellas, recordando el tejido de tipo A de Weiner y Liese (1993) (fig. 2A, D, E). Estas mismas células en corte longitudinal tienen forma isodiamétrica, algunas son grandes y rectangulares, otras cuadradas y de pared delgada (fig. 3E-F). Dentro del segundo tipo del tejido fundamental, se encuentran embebidos numerosos haces fibrosos distribuidos aleatoriamente. Su diámetro tangencial es de 209 (118-284) $\mu \mathrm{m}$ (fig. 2D, F; 3D). Las fibras son también plurilaminadas, cuyo diámetro tangencial de la luz es de $6(1-10) \mu \mathrm{m}$ con una pared de 26 (21-60) $\mu \mathrm{m}$ (fig. 2D, E; 3D). Se presentan células estegmatas esféricas acomodadas en filas discontinuas en la periferia de las fibras de los haces fibrovasculares y fibrosos (fig. 3G). 


\section{Resumen taxonómico}

Etimología. El epíteto específico es en honor al pueblo indígena binacional Kikaapoa, «los que andan por la tierra», localizados en El Nacimiento de los Kikapúes, ubicado en el municipio de Melchor Múzquiz, Coahuila, México.

Holotipo. MUZ-1336

Medidas. $24 \mathrm{~cm}$ de diámetro y $40 \mathrm{~cm}$ de alto.

Localidad. Rancho Atascoso, municipio de Múzquiz, Coahuila, México.

Horizonte y edad. Formación Olmos (Campaniano superior).

\section{Discusión}

\section{Comparación con representantes actuales}

Palmoxylon kikaapoa presenta caracteres propios de la familia Arecaceae, como son: haces fibrovasculares simples embebidos en un tejido fundamental y distribuidos aleatoriamente formando un atactostela (Rich, 1987; Simpson, 2006; Tomlinson y Wilder, 1984). Presenta características anatómicas que se encuentran en 2 subfamilias: Arecoideae y Coryphoideae. Las semejanzas y diferencias con cada una de estas familias se discuten a continuación.

La presencia de haces fibrovasculares con forma reniforme, así como una quilla formada por la unión de los tejidos en el área vascular, permite relacionar taxonómicamente a la palma fósil con la subfamilia Arecoideae (Burnett, 1835; Tomlinson et al., 2011). Con base en características más específicas, $P$. kikaapoa se puede relacionar en particular con 2 tribus de dicha subfamilia. La presencia de un tejido vascular reducido, el floema en un conjunto, elementos del metaxilema con placas de perforación escalariformes y oblicua, así como un tejido fundamental tipo A o aerenquimatoso (según Weiner y Liese, 1993), además de la presencia de estegmatas, hacen que la palma fósil se relacione superficialmente a la tribu Oranieae. Sin embargo, esta tribu se distingue particularmente por presentar un solo vaso de metaxilema, con haces fibrovasculares en el área fibrosa muy desarrollada, presentando fibras masivas, el tejido fundamental aerenquimatoso tiene espacios intercelulares muy grandes con presencia de rafidios, por lo que se descarta por completo alguna relación taxonómica con esta tribu (Tomlinson et al., 2011). Palmoxylon kikaapoa también podría relacionarse con la tribu Areceae, subtribu Arecinae. Las características que hacen comparable a P. kikaapoa con esta tribu son: elementos de vaso del metaxilema con placa de perforación escalariforme y oblicua, elementos del protoxilema con placa de perforación simple, haces fibrovasculares con el área fibrosa, con fibras de pared gruesa arregladas progresivamente, numerosos haces fibrosos en el tejido fundamental y estegmatas de forma esférica. Sin embargo, Arecinae se caracteriza por tener un solo vaso de metaxilema y el tejido fundamental es de tipo lacunoso, lo cual descarta cualquier relación taxonómica del fósil con este grupo (Tomlinson et al., 2011; Uhl y Dransfield, 1987).

Por otra parte, la subfamilia Coryphoideae presenta varias características que se encuentran en $P$. kikaapoa, como son la presencia de haces fibrovasculares en forma reniforme con un área fibrosa bien diferenciada y altamente esclerotizada, elementos de metaxilema con placa de perforación escalariforme y oblicua (Couvreur, Forest y Baker, 2011; Thomas y de Franceschi, 2012; Tomlinson et al., 2011). Los caracteres diagnósticos presentes en el fósil lo relacionan muy estrechamente con la tribu Trachycarpeae: presencia de 2 a 3 (1-4) vasos de metaxilema, los elementos conductores de los haces fibrovasculares rodeados por parénquima paravascular desarrollado con pared delgada y a su vez rodeado por células de parénquima tabular, el floema en un solo conjunto, el área fibrosa sin elongación radial rodeada por parénquima tabular, el tejido fundamental con tendencia a ser aerenquimatoso recordando el tejido de tipo A de acuerdo con Weiner y Liese (1993), embebidos en este tejido fundamental numerosos haces fibrosos distribuidos aleatoriamente, así como la presencia de estegmatas esféricas acomodadas en filas discontinuas en la periferia de las fibras de los haces fibrovasculares y fibrosos (Thomas, 2011; Thomas y de Franceschi, 2012; Tomlinson et al., 2011). En este sentido, $P$. kikaapoa se relaciona más específicamente con el género actual Copernicia Mart. ex Endl., las características que la hacen afín con este género son: presencia de haces fibrovasculares con forma reniforme; 1-4 vasos del metaxilema con placa de perforación escalariforme y oblicua; parénquima paravascular desarrollado; floema en un solo conjunto; parénquima tabular con 2-8 capas de células alargadas; tejido fundamental aerenquimatoso tipo A; numerosos haces fibrosos en el tejido fundamental; estegmatas esféricas en filas discontinuas en la periferia de las fibras de los haces fibrovasculares y fibrosos. Aunque existe una estrecha relación del fósil con este género, hay algunas diferencias anatómicas, por ejemplo, Copernicia presenta parénquima radiado con placas de perforación en el protoxilema no compuestas, aun así, es evidente la estrecha relación anatómica del fósil con Copernicia.

\section{Comparación con especies fósiles}

Palmoxylon kikaapoa presenta haces fibrovasculares y fibrosos embebidos en el tejido fundamental aerenquimatoso tipo A formando un atactostela, con 1 a 4 elementos de metaxilema, además de parénquima tabular, numerosos haces fibrosos, y la presencia de estegmatas permite relacionarla con varias especies de Palmoxylon cretácicas y del Mioceno de EE. UU., México, Argentina y la India.

Respecto al material descrito en el norte de México, Palmoxylon kikaapoa difiere claramente de 4: Palmoxylon longum, $P$. commune, $P$. fibrosum y $P$. polymorphum (tabla 1) de las 5 especies que se han descrito en sedimentos de la Formación Olmos. Las 4 especies citadas anteriormente no presentan un tejido fundamental con espacios intercelulares, además, P. longum presenta placas de perforación simples; por otra parte, $P$. commune, $P$. fibrosum tienen de 2-8 vasos del metaxilema, mientras que $P$. polymorphum hasta más de 10 (CevallosFerriz y Ricalde-Moreno, 1995), descartando alguna afinidad a nivel específico. Recientemente, en los mismos sedimentos se describió una quinta especie, $P$. enochii; esta palma presenta características que la relacionan con la nueva especie aquí descrita. Palmoxylon enochii tiene un tejido fundamental de tipo 
Tabla 1

Comparación de caracteres de especies de Palmoxylon del Cretácico tardío y Mioceno.

\begin{tabular}{llllllll}
\hline Especies & DTVM $(\mu \mathrm{m})$ & $\mathrm{HV} / \mathrm{cm}^{2}$ & $\mathrm{DHV}$ & $\mathrm{MX}$ & TJF & PP & PITVM \\
\hline Palmoxylon simperi & $200-130$ & $?$ & $\mathrm{Al}$ azar & $2-3$ & Compacto & Simples & Escalariformes \\
P. prístina & $120-40$ & $?$ & $\mathrm{Al}$ azar & $2-5$ & Compacto & $?$ & $?$ \\
P. waagei & $70(150-200)$ & $?$ & $\mathrm{Al}$ azar & $2-6$ & Compacto & Simples & $?$ \\
P. longum & $23-38$ & 31 & $\mathrm{Al}$ azar & 2 & Compacto & Simples y transversas & Opuestas y transicionales \\
P. commune & $37(25-55)$ & 48 & $\mathrm{Al}$ azar & $2-8$ & Compacto & $?$ & Escalariformes \\
P. fibrosum & $112(52-160)$ & 83 & $\mathrm{Al}$ azar & $2-8$ & Compacto & $?$ & $?$ \\
P. polymorphum & $51(35-80)$ & 40 & $\mathrm{Al}$ azar & 2 a más de 10 & Compacto & Escalariformes & Escalariformes \\
P. santarosense & 77 & $?$ & $\mathrm{Al}$ azar & $1-2$ & Lacunar & Escalariformes & Escalariformes y opuestas \\
P. riograndense & 52 & $?$ & $\mathrm{Al}$ azar & $4-6$ & Compacto & Escalariformes & Escalariformes \\
P. valchetense & 54 & $?$ & $\mathrm{Al}$ azar & $2-3$ & Compacto & Escalariformes & Escalariformes \\
P. pichaihuensis & $150-200$ & $85-105$ & $\mathrm{Al}$ azar & $2-3$ & Compacto & Simples & Escalariformes \\
P. bhisiensis & $100-200$ & $30-35$ & $\mathrm{Al}$ azar & 2 & Lacunar & Simples & Escalariformes \\
P. enochi & $83(30-150)$ & $17-34$ & Tendencia radial & $2-3$ & Lacunar & Simples & Opuestas \\
P. garridoi & $66(30-113)$ & $?$ & $\mathrm{Al}$ azar & $2-6$ & Lacunar & Simples y escalariformes & $?$ \\
P. romeroi & $79(49-118)$ & $8-16$ & $\mathrm{Al}$ azar & $3-5$ & Compacto & Simples & Alternas \\
P. kikaapoa & $\mathbf{7 4}(\mathbf{4 2 - 1 1 9})$ & $\mathbf{1 3 - 1 9}$ & $\mathrm{Al}$ azar & $\mathbf{2 - 4}$ & Lacunar & Escalariformes & Opuestas a escalariformes \\
\hline
\end{tabular}

DHV: disposición de los haces vasculares; DTVM: diámetro tangencial de los vasos del metaxilema; $\mathrm{HV} / \mathrm{cm}^{2}$ : haces fibrovasculares por centímetro cuadrado; MX: metaxilema; PITVM: punteaduras intervasculares en el metaxilema; PP: placas de perforación; TJF: tejido fundamental.

Negritas: Palmoxylon kikaapoa.

lacunoso, pero la presencia de 2-3 vasos del metaxilema, con 3 a 9 elementos traqueales del protoxilema, así como placa de perforación simple, son una combinación de caracteres que no se encuentran en P. kikaapoa descartando cualquier afinidad (Cevallos-Ferriz y Ricalde-Moreno, 1995; Estrada-Ruiz y Cevallos-Ferriz, 2009) (tabla 1).

En varias localidades de EE. UU. se han estudiado tallos de palmas de edad cretácica (Harley, 2006). Tidwell, Rushforth, Reveal y Behunin (1970) describieron 2 especies de Palmoxylon que en un principio se habían documentado como precretácicas, pero estudios posteriores sobre la geología del lugar propusieron una edad paleógena para estos materiales (Tidwell et al., 1972). Palmoxylon simperi Tidwell, Rushforth, Reveal y Behunin y P. pristina Tidwell, Rushforth, Reveal y Behunin presentan un tejido fundamental tipo compacto, además, P. simperi presenta placas de perforación simple y P. pristina 2-5 vasos del metaxilema, por lo que se descarta alguna relación taxonómica con P. kikaapoa (tabla 1). En la Formación Fox Hills (Maastrichtiano) Cretácico Superior del Western Interior, Dakota del Sur, Delevoryas (1964) describió P. waagei Delevoryas; esta especie presenta un tejido fundamental de tipo compacto, con placa de perforación simple, 2 características que no se encuentran en P. kikaapoa (tabla 1). Recientemente, Estrada-Ruiz, Upchurch, Wheeler y Mack 2012 y Estrada-Ruiz, Parrot, Upchurch, Wheeler, Thompson y Mack (2012) mencionan la presencia de varias angiospermas en sedimentos de la Formación McRae (Campaniano superior) en Nuevo México; del material mostrado, describen informalmente una especie de Palmoxylon, que presenta un tejido fundamental lacunoso, placas de perforación escalariforme, características encontradas en P. kikaapoa, pero difiere de la especie de Nuevo México por la presencia de 2-3 vasos del metaxilema, así como punteaduras intervasculares de tipo escalariforme (Estrada-Ruiz et al., 2012a).
En Argentina se han descrito varias especies cretácicas de Palmoxylon. Ancibor (1995) describió 3 especies de palmas, $P$. riograndense Ancibor, P. santarosense Ancibor, P. valchetense Ancibor del Cretácico tardío, que presentan placas de perforación escalariformes con vasos con un diámetro tangencial promedio pequeño (52 a $77 \mu \mathrm{m})$, en particular $P$. santarosense presenta 1-2 vasos del metaxilema; por otra parte, $P$. riograndense y $P$. valchetense no presentan un tejido fundamental lacunar como $P$. kikaapoa, por lo que se descarta alguna relación taxonómica (tabla 1). Ottone (2007) describe una palmera del Cretácico tardío de Argentina, P. pichaihuensis Ottone, esta palma presenta 1-5 vasos de metaxilema con placas de perforación simples y punteaduras intervasculares de tipo escalariforme, además, un tejido fundamental compacto, son caracteres que no comparte con la nueva especie fósil descrita de la Formación Olmos. Martínez (2012) describe una especie que presenta caracteres encontrados en P. kikaapoa como son un tejido fundamental de tipo lacunar y placas de perforación escalariforme. Sin embargo, la presencia de haces fibrovasculares del tipo Antiguensia sensu Stenzel, además de 2-6 vasos del metaxilema con placas escalariformes y simples descartan alguna relación taxonómica con P. garridoi (Martínez, 2012). Recientemente, en sedimentos del Mioceno superior en la Formación Chiquimil del Grupo Santa María en Argentina, Franco, Brea y Herbst (2014) describieron una especie de Palmoxylon dentro de la subfamilia Coryphoideae, de la tribu Cryosophileae; este nuevo registro presenta caracteres que no permiten relacionarlo con el ejemplar mexicano como son la presencia de 3 a 5 vasos del metaxilema con placas de perforación simples.

Finalmente, Dutta, Ambwani y Prasad (2007) en sedimentos cretácicos de la Formación Lameta, India, describieron a Palmoxylon bhisiensis Dutta, Ambwani et Prasad, una palma con tejido fundamental lacunar, pero que difiere de $P$. kikaapoa porque presenta placas de perforación simples y la forma de las 
células en su tejido fundamental son distintas con el ejemplar mexicano (tabla 1).

Es claro que Palmoxylon kikaapoa de la Formación Olmos (Cretácico Superior, Campaniano superior) presenta características únicas no encontradas en otras palmas cretácicas; por tal motivo se propone una nueva especie dentro de la subfamilia Coryphoideae para el Cretácico Superior del norte de México. Representantes actuales de la subfamilia Coryphoideae presentan una distribución principalmente pantropical y subtropical. La tribu Trachycarpeae es la más grande de las tribus de esta subfamilia y sus centros principales de distribución son EE: UU. y el Caribe y en los ambientes tropicales del este de Asia; está ausente en África (Bacon, 2011; Dransfield et al., 2008; Tomlinson et al., 2011). En particular, el género Copernicia está representado por 21 especies, de las cuales 3 se distribuyen en Sudamérica, 2 en el Caribe y el resto en Cuba; los cuerpos vegetativos de estas palmas son arbóreos como plantas únicas y raramente en agregados (Dransfield et al., 2008; Tomlinson et al., 2011). Es probable que la especie fósil no haya formado agregaciones, debido a que esta planta crecía muy cerca de los cuerpos de agua como lo indica su anatomía aerenquimatosa.

Este nuevo fósil de palma representa el registro más antiguo para la subfamilia Coryphoideae, tribu Trachycarpeae para América del Norte, siendo así otro aporte de importancia para el conocimiento de la biodiversidad de las floras del Cretácico Superior del norte de México. Con base en el registro fósil y estudios filogenéticos, se ha propuesto que la subfamilia Coryphoideae se originó en el Hemisferio Norte, posteriormente se diversificó siguiendo una ruta boreotropical hacia América Central y América del Sur durante el Cretácico Superior y Cenozoico (Bjorholm, Svenning, Baker, Skov y Balslev, 2006; Dransfield et al., 2008; Morley, 2000). Además, por la distribución de las especies actuales de Trachycarpeae se propone un origen Laurásico (Bacon, 2011; Baker et al., 2009; Dransfield, 1987; Dransfield et al., 2008); este nuevo registro de la Formación Olmos concuerda y soporta las hipótesis propuestas, indicando que durante el Cretácico Superior, Coryphoideae fue un elemento importante en estas floras cretácicas de EE. UU., donde estaba representada por elementos arbóreos. Dicho hábito es apoyado por otros registros de palmas fósiles y de otras maderas de dicotiledóneas representadas por árboles como Javelinoxylon (Malvaceae s.1.), Sabinoxylon Estrada-Ruiz, Martínez-Cabrera y Cevallos-Ferriz (Ericales), Quercinium Unger (Fagaceae), Coahuilaxylon Estrada-Ruiz, Martínez-Cabrera y Cevallos-Ferriz (Anacardiaceae/Burseraceae), Muzquizoxylon Estrada-Ruiz, Martínez-Cabrera y Cevallos-Ferriz (Cornaceae), Olmosoxylon Estrada-Ruiz, Martínez-Cabrera y Cevallos-Ferriz (Lauraceae) y Metcalfeoxylon (incertae sedis) que llegaron a formar varios estratos arbóreos dentro de la selva paratropical (Estrada-Ruiz et al., 2013; Wheeler y Lehman, 2000). En particular, uno de los troncos pertenecientes a Javelinoxylon presentó una longitud de $14 \mathrm{~m}$ y ca. $80 \mathrm{~cm}$ de diámetro. Lehman y Wheeler (2001) mencionan que este mismo género y Metcalfeoxylon fueron árboles grandes que formaron distintos estratos arbóreos como en las Formaciones Aguja en Texas y, Kirkland y Crevasse Canyon en Nuevo México (Estrada-Ruiz et al., 2012a; EstradaRuiz et al., 2012b; Wheeler, McClamer y LaPasha, 1995); algunas de estas plantas llegaron a crecer de 30 a $40 \mathrm{~m}$ de altura.

Una de las características de los bosque tropicales húmedos es la gran abundancia de palmas (Kahn y de Granville, 1992). A pesar de que generalmente es aceptado que este bioma es relativamente antiguo, la ubicación y el momento de su origen se mantienen inciertos, principalmente porque el registro fósil es incompleto, especialmente durante el Cretácico. Sin embargo, registros paleobotánicos previos indican que en la Formación Olmos se desarrolló una flora que creció entre los 21 a $24{ }^{\circ} \mathrm{C}$, representando hasta el momento la selva paratropical más húmeda y diversa que las demás floras del centro-sur de EE. UU. (Estrada-Ruiz et al., 2008, 2013). El registro de $P$. kikaapoa en esta flora cretácica permite reforzar la hipótesis de la presencia de un ambiente tropical en la Formación Olmos, así como proveer de nueva información sobre Trachycarpeae, su diversificación y su distribución en el presente y en el pasado.

\section{Agradecimientos}

Los autores agradecen de una manera especial a las autoridades del municipio de Melchor Múzquiz y a los miembros de PASAC (Paleontólogos Aficionados de Sabinas, A.C.), en especial al Sr. H. Porras-Múzquiz, Director del Museo de Múzquiz, Coahuila todo el apoyo durante nuestra visita al campo. Al Instituto Nacional de Antropología e Historia de Coahuila por las facilidades prestadas para nuestra investigación. A los revisores y editor por los comentarios que mejoraron el trabajo. A Jaime Díaz del Instituto de Geología,UNAM por la realización de las láminas delgadas. A J. Ocaña, M. Sánchez y G. Villegas de la Escuela Nacional de Ciencias Biológicas, IPN, por el préstamo de los microscopios. Parte del trabajo fue financiado por el proyecto SIP y 20150435 del IPN y Conacyt (240241) de EER.

\section{Referencias}

Ancibor, E. (1995). Palmeras fósiles del Cretácico Tardío de la Patagonia Argentina (Bajo de Santa Rosa, Río Negro). Ameghiniana, 32, 287-299.

Angiosperm Phylogeny Group. (2009). An update of the Angiosperm Phylogeny Group classification for the orders and families of flowering plants: APG III. Botanical Journal of the Linnean Society, 161, 106-121.

Bacon, C. D. (2011). Trachycarpeae palms as models to understand patterns of island biogeography and diversification. Tesis doctoral. Fort Collins, Colorado: Colorado State University, EUA. Tesis doctoral

Baker, W. J., Savolainen, V., Asmussen-Lange, C. B., Chase, M. W., Dransfield, J., Forest, F., et al. (2009). Complete generic-level phylogenetic analyses of palms (Arecaceae) with comparison of superthree and supermatrix approaches. Society of Systematic Biologists, 58, 240-256.

Berry, E. W. (1916). A petrified palm from the Cretaceous of New Jersey. American Journal of Science, 41, 193-197

Bjorholm, S., Svenning, J. C., Baker, W. J., Skov, F. y Balslev, H. (2006). Historical legacies in the geographical diversity patterns of New World palm (Arecaceae) subfamilies. Botanical Journal of the Linnean Society, 151, $113-125$.

Brea, M., Zucol, A. F. y Franco, M. J. (2013). Paleoflora de la Formación Paraná (Mioceno tardío), Cuenca Chaco-Paranaense, Argentina. En D. Brandoni y J. I. Noriega (Eds.), El Neógeno de la Mesopotamia argentina (pp. 28-40). Buenos Aires: Asociación Paleontológica Argentina. Publicación Especial 14. 
Burnett, G. T. (1835). Outlines of botany, including a general history of the vegetable kingdom, in which plants are arranged according to the system of natural affinities. London: H. Renshaw.

Cevallos-Ferriz, S. R. S. (1992). Tres maderas de gimnospermas cretácicas del norte de México. Anales Instituto de Biología, Universidad Nacional Autónoma de México, 63, 111-137.

Cevallos- Ferriz, S. R. S. y Ricalde-Moreno, O. S. (1995). Palmeras fósiles del norte de México. Anales del Instituto de Biología, Universidad Nacional Autónoma de México, Serie Botánica, 66, 37-106.

Cevallos-Ferriz, S. R. S. y Weber, R. (1992). Dicotyledoneous wood from the upper Cretaceous (Maastrichtian) of Coahuila. Universidad Nacional Autónoma de México, Instituto de Geología, Revista, 10, 65-70.

Chase, M. W. y Reveal, J. L. (2009). A phylogenetic classification of the land plants to accompany APG III. Botanical Journal of the Linnean Society, 161, $122-127$.

Couvreur, T. L. P., Forest, F. y Baker, W. J. (2011). Original and global diversification patterns of tropical rain forests: Inferences from a complete genus-level phylogeny of palms. BMC Biology, 9, 44.

Delevoryas, T. (1964). Two petrified angiosperms from the upper Cretaceous of South Dakota. Journal of Paleontology, 38, 584-586.

Dransfield, J. (1987). Bicentric distribution in Malesia as exemplified by palms. En T. C. Whitmore (Ed.), Biogeographical evolution of the Malay Archipelago (pp. 60-70). Oxford: Clarendon Press.

Dransfield, J., Uhl, N. W., Assmussen, C. B., Baker, W. J., Harley, M. M. y Lewis, C. L. (2008). Genera Palmarum: the evolution and classification of Palms. London: Kew Publishing. Royal Botanical Gardens.

Dutta, D., Ambwani, K. y Prasad, M. (2007). Occurrence of upper-Cretaceous fossil palm wood, Palmoxylon bhisiensis sp. nov. from the Lameta Formation of Bhisi village, Maharashtra, India. Journal of Applied of Bioscience, 33, $8-13$.

Estrada-Ruiz, E. (2009). Reconstrucción de los ambientes de depósito y paleoclima de la región de Sabinas-Saltillo, estado de Coahuila, con base en plantas fósiles del Cretácico Superior. Instituto de Geología, Universidad Nacional Autónoma de México. Tesis doctoral.

Estrada-Ruiz, E. y Cevallos-Ferriz, S. R. S. (2009). Palmoxylon enochii sp. nov. de la Formación Olmos (Campaniano superior-Maastrichtiano inferior), Coahuila, México. Ameghiniana, 46, 577-585

Estrada-Ruiz, E. y Martínez-Cabrera, H. I. (2011). A new late Cretaceous (Coniacian-Maastrichtian) Javelinoxylon wood from Chihuahua, Mexico. IAWA Journal, 32, 519-528.

Estrada-Ruiz, E., Martínez-Cabrera, H. I., Callejas-Moreno, J. y Upchurch, G. R., Jr. (2013). Floras tropicales cretácicas del norte de México y su relación con floras del centro-sur de América del Norte. Polibotánica, 36, 41-61.

Estrada-Ruiz, E., Martínez-Cabrera, H. I. y Cevallos-Ferriz, S. R. S. (2007). Fossil wood from the late Campanian-early Maastrichtian Olmos Formation, Coahuila, Mexico. Review of Palaeobotany and Palynology, 45, $123-133$.

Estrada-Ruiz, E., Martínez-Cabrera, H. I. y Cevallos-Ferriz, S. R. S. (2010). Fossil wood from the Olmos Formation (late Campanian-early Maastrichtian) Coahuila, Mexico. American Journal of Botany, 97, 1179-1194.

Estrada-Ruiz, E., Parrot, J. M., Upchurch, G. R. Jr., Wheeler, E. A., Thompson, D. L., Mack, G. et al. (2012). The wood flora from the upper Cretaceous Crevasse Canyon and McRae Formations, South-central New Mexico, USA: a progress report. Sierra County: field guide of New Mexico Geological Society, 63rd Field Conference.

Estrada-Ruiz, E., Upchurch, G. y Cevallos-Ferriz, S. R. S. (2008). Flora and climate of the Olmos Formation (upper Campanian-lower Maastrichtian), Coahuila, Mexico. A preliminary report. Gulf Coast Association of Geological Societies Transactions, 58, 273-283.

Estrada-Ruiz, E., Upchurch, G. R., Jr., Wheeler, E. A. y Mack, G. (2012). Late Cretaceous angiosperm woods from the Crevasse Canyon and McRae Formations, South-central New Mexico, USA: part 1. International Journal of Plant Sciences, 173, 412-428.

Estrada-Ruiz, E., Upchurch, G. R., Jr., Wolfe, J. A. y Cevallos-Ferriz, S. R. S. (2011). Comparative morphology of fossil and extant leaves of Nelumbonaceae, including a new genus from the late Cretacceous of Western North America. Systematic Botany, 32, 337-351.
Franco, M. J., Brea, M. y Herbst, R. (2014). Palmoxylon romeroi sp. nov., de la Formación Chiquimil (Mioceno Superior) del Valle de Santa María, Provincia de Catamarca, Argentina. Ameghiniana, 51, 572-584.

Haas, H. y Rowe, N. P. (1999). Thin sections and wafering. En T. P. Jones y N. P. Rowe (Eds.), Fossil plants and spores: modern techniques (pp. 76-81). London: Geological Society.

Harley, M. M. (2006). A summary of fossil record for Arecaceae: Botanical Journal of the Linnean Society, 151, 39-67.

Kahn, F. y de Granville, J. J. (1992). Palms in forest ecosystems of Amazonia. Berlín: Springer Verlag.

Lehman, T. M. y Wheeler, E. A. (2001). Fossil dicotyledonous forest from the upper Cretaceous of Big Bend National Park, Texas. Palaios, 16, 102-108.

Macko, Z. (1957). Lower Miocene pollen flora from the valley of Keodnicka near Gliwice (upper Silesia). Prace Wroclawskiego Towarzystwa Naukowego, Ser, 13, 1-313.

Martínez, L. C. A. (2012). Estípite de palmera en el Campaniano del grupo Neuquén, provincia del Neuqué, Argentina. Ameghiniana, 49, 2573-2584.

Morley, R. (2000). Origin and evolution of tropical rainforests. Chichester: Wiley.

Ottone, E. G. (2007). A new palm trunk from the upper Cretaceous of Argentina. Ameghiniana, 44, 719-725.

Palmweb. (2011). Palmweb: Palms of the World Online. Recuperado: 30 de enero de 2015 de: http://www.palmweb.org

Rich, P. M. (1987). Developmental anatomy of the stem of Welfia Georgii, Iriartea gigantea and other arborescent palms: implications for mechanical support. American Journal of Botany, 74, 792-802.

Sahni, B. (1964). Revisions of Indian fossil plants. Part III Monocotyledons Lucknow: Birbal Sahni Institute of Palaeobotany.

Schenk, A. (1882). Die von den Gebrüdern Schlagenwelt in Indien gesammelten fossilen Hölzer. Botanischer Jahrbücher fur Systematik, Planzengeschichte und Planzengeographie, 3, 353-358.

Serlin, B., Delevoryas, T. y Weber, R. (1981). A new conifer pollen cone from the upper Cretaceous of Coahuila, Mexico. Review of Palaeobotany and Palynology, 31, 241-248.

Simpson, M. G. (2006). Plant sistematics. Burlington, Massachusetts: Elsevier, Academic Press.

Thomas, R. (2011). Palm-ID, a database to identify the palm stem anatomy with an expert system (Xper 2). Paris: Université Paris 6 , Muséum National d'Histoire Naturelle. Recuperado: enero de 2015 de: http://www.infosyslab.fr/Palm-ID/

Thomas, R. y de Franceschi, D. (2012). First evidence of fossil Cryosophileae (Arecaceae) outside the Americas (early Oligocene and late Miocene of France): anatomy, palaeobiogeography and evolutionary implications. Review of Palaeobotany and Palynology, 171, 27-39.

Thomas, R. y de Franceschi, D. (2013). Palm stem anatomy and computer-aided identification: the Coryphoideae (Arecaceae). American Journal of Botany, 100, 289-313.

Tidwell, W. D., Rushforth, J. L., Reveal, J. L. y Behunin, H. (1970). Palmoxylon simperi and Palmoxylon pristina: two pre-Cretaceous angiosperms from Utah. Science, 168, 835-840.

Tidwell, W. D., Medlyn, D. A. y Thyan, G. F. (1972). Fossil palm materials from the Tertiary Dipping Vat Formation of Central Utah. The Great Basin Naturalist, 32, 1-15

Tomlinson, P. B., Horn, J. W. y Fisher, J. B. (2011). The anatomy of palms Oxford: Oxford University Press.

Tomlinson, P. B. y Wilder, G. J. (1984). Systematic anatomy of Cyclanthaceae (Monocotyledoneae)-an overview. Botanical Gazette, 145, 535-549.

Uhl, N. W. y Dransfield, J. (1987). Genera Palmarum, a classification of the palms based on the work of Harold E. Moore Jr. Lawrence. Liberty Hyde Bailey Hortorium and the International Palm Society.

Weber, R. (1972). La vegetación maestrichtiana de la Formación Olmos de Coahuila, México. Boletín de le Sociedad Geológica Mexicana, 33, 5-19

Weber, R. (1973). Salvinia coahuilensis nov. sp. del Cretácico superior de México. Ameghiniana, 10, 173-190.

Weber, R. (1975). Aachenia knoblochi n. sp. an interesting conifer of the upper Cretaceous Olmos Formation of Northeastern Mexico. Palaeontographica Abteilung B, 152, 76-83. 
Weber, R. (1976). Dorfiella auriculata f. gen. nov., sp. nov. Un género nuevo de helechos acuáticos del Cretácico Superior de México. Boletín de la Asociación Latinoamericana de Paleobotánica y Palinología, 3, $1-13$.

Weber, R. (1978). Some aspects of the upper Cretaceous angiosperm flora of Coahuila, Mexico. Courier Forchungs-Institut Senckenberg, 30, $38-46$.

Weiner, G. y Liese, W. (1993). Generic identification key to rattan palms based on stem anatomical characters. IAWA Journal, 14, 55-61.
Wheeler, E. A. y Lehman, T. M. (2000). Late Cretaceous woody dicots from the Aguja and Javelina Formations, Big Bend National Park, Texas. IAWA Journal, 21, 83-120.

Wheeler, E. A., Lehman, T. M. y Gasson, P. (1994). Javelinoxylon, a new genus of malvalean tree from the upper Cretaceous of Big Bend National Park, Texas. American Journal of Botany, 81, 703-710.

Wheeler, E. A., McClamer, J. y LaPasha, C. A. (1995). Similarities and differences in dicotyledonous woods of the Cretaceous and Paleocene. San Juan Basin, New Mexico, USA. IAWA Journal, 16, 223-254. 